\title{
Coassociations between IL 10 polymorphisms, IL-10 production, helminth infection, and asthma/wheeze in an urban tropical population in Brazil
}

\author{
Camila Alexandrina Figueiredo, PhD, ${ }^{\text {a,b }}$ Maurício Lima Barreto, MD, PhD, ${ }^{c}$ Neuza Maria Alcantara-Neves, MD, PhD, \\ Laura Cunha Rodrigues, MD, PhD, ${ }^{d}$ Philip John Cooper, MD, PhD, , f Alvaro A. Cruz, MD, PhD, ${ }^{g}$

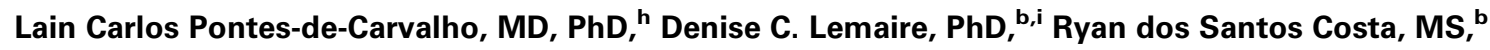 \\ Leila D. Amorim, PhD, ${ }^{j}$ Candelaria Vergara, MD, PhD, ${ }^{a}$ Nicholas Rafaels, MS, ${ }^{a}$ Li Gao, MD, PhD, ${ }^{a}$ Cassandra Foster, ${ }^{a}$ \\ Monica Campbell, BS, ${ }^{a}$ Rasika A. Mathias, ScD, ${ }^{\text {a }}$ and Kathleen C. Barnes, PhD ${ }^{\mathbf{a}}$ Baltimore, Md, Salvador, Brazil, London, \\ United Kingdom, and Quito, Ecuador
}

Background: Helminth infections are associated with protection against allergies. It is postulated that IL-10 production after helminth infection suppresses skin hypersensitivity and increases $\mathrm{IgG}_{4}$ production, protecting against allergies. Objective: We aimed to determine whether IL10 polymorphisms are associated with helminth infection and the risk of wheeze and allergy.

Methods: Twelve IL10 single nucleotide polymorphisms were genotyped in 1353 children aged 4 to 11 years living in a poor urban area in Salvador, Brazil. Wheezing status, Ascaris lumbricoides and Trichuris trichiura infection, IL-10 production by peripheral blood leukocytes stimulated with $A$ lumbricoides

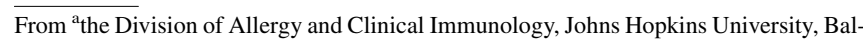
timore; ' Instituto de Ciências da Saúde, ${ }^{\mathrm{C}}$ Instituto de Saúde Coletiva, ${ }^{\mathrm{g}}$ ProAR-Núcleo de Excelência em Asma, and ${ }^{\mathrm{j}}$ Instituto de Matemática, Universidade Federal da Bahia, Salvador; ${ }^{\mathrm{d}}$ the London School of Hygiene and Tropical Medicine; ${ }^{\mathrm{e}}$ Universidad San Francisco de Quito; fthe Centre for Infection, St George's, University of London; ${ }^{\mathrm{h}}$ Centro de Pesquisas Gonçalo Moniz, Fundação Oswaldo Cruz-FIOCRUZ, Salvador; and ${ }^{i}$ Departamento de Ciências da Vida, Universidade do Estado da Bahia, Salvador. Supported by the Wellcome Trust, UK; the HCPC Latin America Excellence Centre Programme, Ref 072405/Z/03/Z; the Brazilian agency Conselho Nacional de Desenvolvimento Científico e Tecnológico-CNPq; and the David G. Marsh Award. K.C.B. was supported in part by the Mary Beryl Patch Turnbull Scholar Program.

Disclosure of potential conflict of interest: M. L. Barreto has received grants from the Wellcome Trust and the National Research Council-Brazil and is employed by the Federal University of Bahia. P. J. Cooper has received grants from the Wellcome Trust and is employed by the Liverpool School of Tropical Medicine. A. A. Cruz serves on boards for Merck Sharp \& Dohme and Roche; has consultant arrangements with Mantecorp; receives grants from GlaxoSmithKline; receives payment for lectures from Merck Sharp \& Dohme; has received travel/accommodations/meeting expenses from Merck Sharp \& Dohme and Novartis; and has conducted clinical trials for AstraZeneca, Novartis, TAKEDA, Eurofarma, GlaxoSmithKline, and Genentech. L. C. Pontes-de-Carvalho is employed by the Ministry of Health, Brazil. C. Vergara, C. Foster, M. Campbell, and R. A. Mathias are employed by Johns Hopkins University and have received grants from the National Institutes of Health. K. C. Barnes serves on boards for Genentech; has consultant arrangements with Sanofi-Aventis and Sirius Genomics; is employed by Johns Hopkins University; has received grants from the National Institutes of Health/National Heart, Lung, and Blood Institute; has received payment for lectures from the "Evolution and Diseases of Modern Environments" Symposium, the Cincinnati Children's Hospital Medical Center Allergy Conference, the 50th Annual Swineford Allergy Conference, and the American Academy of Allergy, Asthma \& Immunology; and receives royalties from Up-To-Date. The rest of the authors declare that they have no relevant conflicts of interest.

Received for publication June 20, 2012; revised October 16, 2012; accepted for publication October 19, 2012

Available online December 28, 2012.

Corresponding author: Kathleen C. Barnes, PhD, Division of Allergy and Clinical Immunology, Johns Hopkins University, Baltimore, MD. E-mail: kbarnes@jhmi.edu. $0091-6749 / \$ 36.00$

(C) 2012 American Academy of Allergy, Asthma \& Immunology

http://dx.doi.org/10.1016/j.jaci.2012.10.043 extract, serum total IgE levels, specific IgE levels, skin prick test responses to common aeroallergens, and $\mathrm{IgG}_{4}$ and $\mathrm{IgE}$ anti-A lumbricoides antibody levels were measured in all children. Association tests were performed by using logistic or linear regression when appropriate, including sex, age, helminth infection, and principal components for ancestry informative markers as covariates by using PLINK.

Results: Allele $G$ of marker rs3024496 was associated with the decreased production of IL-10 by peripheral blood leukocytes in response to $A$ lumbricoides stimulation. Allele $\mathrm{C}$ of marker rs3024498 was negatively associated with helminth infection or its markers. Marker rs3024492 was positively associated with the risk of atopic wheeze, total IgE levels, and skin prick test responses to cockroach.

Conclusions: Our findings suggest that $I L 10$ polymorphisms might play a role in the production of IL-10, helminth infection, and allergy. We hypothesize that polymorphisms related to protection against helminths, which would offer an evolutionary advantage to subjects in the past, might be associated with increased risk of allergic diseases. (J Allergy Clin Immunol 2013;131:1683-90.)

Key words: IL10, polymorphisms, helminth infection, immune modulation, allergy, asthma, Social Changes Asthma and Allergy in Latin America

The incidence of chronic inflammatory disorders, including allergic disorders (asthma and hay fever) and autoimmune disease (type 1 diabetes and multiple sclerosis), has been increasing markedly on a global level. ${ }^{1}$ The hygiene hypothesis attributes some of these increases to a failure of immunoregulation caused by diminished exposure to certain organisms that have coexisted with definitive hosts throughout their mammalian evolutionary history. $^{2}$

Helminths are multicellular parasites that infect more than 2 billion persons around the world ${ }^{3}$ but are steadily being eliminated in westernized societies. Most helminths are well tolerated, and most carriers are clinically asymptomatic. ${ }^{3}$ A special hostparasite relationship has developed over time, such that some species have the ability to live for decades in the human host through specially developed mechanisms of activation and regulation of immune responses that guarantee homeostasis within the host. ${ }^{4}$

We and others have previously demonstrated that parasites, such as Ascaris lumbricoides and Trichuris trichiura, can modulate immune response inducing hyporesponsiveness of peripheral 


\author{
Abbreviations used \\ CEU: Utah residents with Northern and Western European ancestry \\ from the CEPH population \\ DAG: Direct acyclic graph \\ LD: Linkage disequilibrium \\ SNP: Single nucleotide polymorphism \\ SPT: Skin prick test \\ YRI: Yoruban in Ibadan, Nigeria
}

blood leukocytes to mitogen stimulation through activation of a regulatory network ${ }^{5,6}$ by increasing the production of the regulatory cytokine IL-10. In the same study population we have also shown that children who live in poor areas are more likely to be infected with A lumbricoides and T trichiura and produce IL-10 in culture. ${ }^{7}$

In the present work we sought to explore genetic associations between IL10 polymorphisms, IL-10 production, helminth infection, asthma/wheezing, and markers of allergy in a population of children living in poor urban areas of Brazil to determine whether upregulation of IL-10 in this Latin population is due to solely environmental exposures or whether genetic susceptibility is also contributing to the risk of parasitic and allergic disease.

\section{METHODS}

\section{Study population and design}

This study was conducted in the city of Salvador in northeastern Brazil, with a population of 2.5 million. The general study design has been reported elsewhere. ${ }^{5,7-9}$ In short, the study population included 1445 unrelated children between 4 and 11 years old who were recruited in infancy for a prospective study measuring the effect of a citywide sanitation program on childhood morbidity. ${ }^{10}$ Ethical approval for the study was obtained from the Brazilian National Ethical Committee, and written informed consent was obtained from the legal guardian of each child. Data were collected from children born between 1994 and 2001 who lived in sentinel neighborhoods in the city. Standardized questionnaires were administered to the children's guardians between 1997 and 2003 (baseline) to collect data on demographic and social variables, as well as on the home environment. In 2000, stool samples were collected to characterize intestinal helminth infection. Children were surveyed again in 2005 to collect the same variables and data on asthma by using a Portuguese-adapted phase II International Study of Asthma and Allergies in Childhood questionnaire and to obtain stool and blood samples.

\section{Cell culture}

Venous blood was collected into heparinized tubes and cultured at a dilution of 1:4 in RPMI (Gibco, Auckland, New Zealand) containing $10 \mathrm{mmol} / \mathrm{L}$ glutamine (Sigma-Aldrich, St Louis, Mo) and $100 \mu \mathrm{g} / \mathrm{mL}$ gentamicin (Sigma-Aldrich). Cells were cultured within 6 hours of collection in the presence or not of $10 \mu \mathrm{g} / \mathrm{mL}$ endotoxin-free A lumbricoides antigen and were maintained in a humidified environment of $5 \% \mathrm{CO}_{2}$ at $37^{\circ} \mathrm{C}$ for 24 hours before the supernatants were collected for IL-10 measurement.

\section{IL-10 measurement using ELISA}

The IL-10 concentration was measured in whole-blood culture supernatants by using commercially available antibody pairs and recombinant cytokine standards (BD PharMingen, San Diego, Calif) by means of sandwich ELISA, according to the manufacturer's instructions. Cytokine concentrations were determined by means of interpolation of standard curves. Detection limits (low/high) were $31.25 / 500 \mathrm{pg} / \mathrm{mL}$. A total of 1356 children were assayed for IL-10 levels.

\section{Genotyping}

Twelve IL10 single nucleotide polymorphisms (SNPs) were selected for genotyping. A tagging approach suggested SNPs captured $100 \%$ of the genetic variation in an approximately $4.89 \mathrm{Kbp}$ region on chromosome 1 in the European ancestry CEPH (CEU) and African (Yoruban in Ibadan, Nigeria) YRI populations in HapMap (http://hapmap.ncbi.nlm.nih.gov/). In addition, 7 SNPs with prior associations with related phenotypes (rs1800871, rs1800872, rs1800896, rs3024491, rs3024492, rs3024495, and rs3024496) were included, even though they were in high linkage disequilibrium (LD) with the 5 tag SNPs selected. DNA was extracted from peripheral blood samples by using commercial standard protocols (Gentra Puregene Blood Kit; Qiagen, Hilden, Germany). SNPs were typed by using the TaqMan probebased, $5^{\prime}$ nuclease assay minor groove binder chemistry ${ }^{11}$ on the $7900 \mathrm{HT}$ Sequence Detection System (Applied Biosystems, Foster City, Calif). TaqMan-validated assays and master mix were manufactured by Applied Biosystems. PCR was conducted in a $5-\mu \mathrm{L}$ volume by using a universal master mix and 4 predesigned and validated TaqMan assays for the SNPs (list of SNPs is shown in Table I). The thermal cycling conditions were as follows: $95^{\circ} \mathrm{C}$ for 10 minutes, followed by 40 cycles of $95^{\circ} \mathrm{C}$ for 15 seconds $/ 60^{\circ} \mathrm{C}$ for 1 minute and an extension step of $60^{\circ} \mathrm{C}$ for 5 minutes. Nontemplate negative and genotyping-positive controls were included in each genotyping plate. Automatic calling was performed with a quality value of greater than $99 \%$.

Ten percent of the samples were genotyped in duplicate with $100 \%$ reproducibility. All 12 SNPs were in Hardy-Weinberg equilibrium. Allele frequencies of the 12 SNPs are summarized in Table I. Fig 1 depicts SNP localization in the $I L 10$ gene (chromosome 1, approximately $4.89 \mathrm{Kbp}$ ) and the corresponding LD plot showing that all SNPs are in strong LD (ie, strongly correlated, except for markers rs30244898, rs3024505, and rs1800896).

\section{Skin prick tests}

Skin prick tests (SPTs) were performed on the right forearms of each child by using standardized extracts (ALK-Abelló, São Paulo, Brazil) of Dermatophagoides pteronyssinus, Blomia tropicalis, Blattella germanica, Periplaneta americana, fungi, and cat and $\operatorname{dog}$ dander. Saline and 10 $\mathrm{mg} / \mathrm{mL}$ histamine solution were used as negative and positive controls, respectively. Reactions were read after 15 minutes, and a mean wheal size of at least $3 \mathrm{~mm}$ greater than that elicited by the negative control was considered positive.

\section{Specific IgE}

Determination of specific IgE serum concentrations was performed for $D$ pteronyssinus, $B$ tropicalis, $B$ germanica, and $P$ americana by using the ImmunoCAP assay (Phadia Diagnostics AB, Uppsala Sweden). Children with $0.70 \mathrm{kU} / \mathrm{L}$ or greater of specific $\mathrm{IgE}$ for any allergen tested were considered to have positive results.

\section{Wheezing definition}

As previously described, ${ }^{12}$ children were classified as having current wheeze by using phase II International Study of Asthma and Allergies in Childhood questionnaire data (wheezing in the last 12 months) and were considered to have current wheeze plus symptoms if parents reported wheezing in the previous 12 months and at least 1 of the following: (1) diagnosis of asthma ever; (2) wheezing with exercise in the last 12 months; (3) 4 or more episodes of wheezing in the last 12 months; and (4) waking up at night because of wheezing in the last 12 months. We defined wheeze phenotypes (atopic and nonatopic) according to having a positive result $(\geq 0.70 \mathrm{kU} / \mathrm{L})$ or not for at least 1 specific IgE to aeroallergens.

\section{Parasitological analysis}

Duplicate stool samples from each child were collected and analyzed for common parasites at each of the 2 sampling times 2 weeks apart. Stool samples were analyzed by using the gravitational sedimentation technique of Hoffman et $\mathrm{al}^{13}$ to detect helminth eggs, protozoan cysts, and oocysts. Two slides were 
TABLE I. SNPs included in this study

\begin{tabular}{lcllll}
\hline Marker & $\begin{array}{c}\text { Chromosome } \\
\text { position }\end{array}$ & Gene location & Allele & MAF & HWE \\
\hline rs1518111 & 206944645 & Intronic & T/C & 0.365 & 0.257 \\
rs1554286 & 206944233 & Intronic & A/G & 0.344 & 0.215 \\
rs1800871 & 206946634 & $5^{\prime}$ UTR & A/G & 0.376 & 0.207 \\
rs1800872 & 206946407 & 5'UTR & T/G & 0.377 & 0.182 \\
rs1800896 & 206946897 & 5'UTR & C/T & 0.335 & 0.407 \\
rs1878672 & 206943713 & Intronic & C/G & 0.316 & 1 \\
rs3024491 & 206945046 & Intronic & A/C & 0.311 & 0.812 \\
rs3024492 & 206944112 & Intronic & A/T & 0.074 & 0.795 \\
rs3024495 & 206942413 & Intronic & T/C & 0.071 & 0.578 \\
rs3024496 & 206941864 & Exomic & G/A & 0.38 & 0.724 \\
rs3024498 & 206941529 & 3'UTR & C/T & 0.175 & 0.804 \\
rs3024505 & 206939904 & Downstream & A/G & 0.086 & 0.053 \\
\hline
\end{tabular}

$H W E$, Hardy-Weinberg equilibrium; MAF, minor allele frequency; UTR, untranslated region.

examined for each sample. Quantification of helminth eggs was performed by using the Kato-Katz technique. ${ }^{14}$ All children with positive results were treated with appropriate antiparasitic drugs. ${ }^{8}$ Occurrence and chronicity of infections with A lumbricoides and T trichiura were defined as follows: (1) current infections, infections with either parasite detected only later in childhood (ie, survey conducted in 2005); (2) chronic infections, children infected with either A lumbricoides or T trichiura both early in childhood (ie, survey conducted in 2000) and later in childhood (2005); and (3) coinfections, children infected with both helminths in 2005 .

\section{Total lgE level and markers of infection: $\lg E$ and $\lg _{4}$ anti-A lumbricoides antibodies}

Determination of specific IgE serum concentrations was performed for A lumbricoides by using the ImmunoCAP assay (Phadia Diagnostics AB, Uppsala, Sweden). Children with $0.35 \mathrm{kU} / \mathrm{L}$ or greater of anti-A lumbricoides-specific IgE were considered to have positive results.

Anti-A lumbricoides $\mathrm{IgG}_{4}$ was detected by using indirect ELISA as follows. High-binding microassay plate wells (Costar, Cambridge, Mass) were sensitized with $20 \mu \mathrm{g} / \mathrm{mL}$ A lumbricoides antigen diluted in carbonatebicarbonate ( $\mathrm{pH} 9.6$ ) buffer. Sera were diluted 1:50 in $0.15 \mathrm{~mol} / \mathrm{L} \mathrm{PBS}(\mathrm{pH}$ 7.2) containing 10\% FBS (Sigma Chemical Co, St Louis, Mo) and $0.1 \%$ Tween 20. Plates were incubated with biotinylated anti-human $\operatorname{IgG}_{4}$ (Sigma Chemical Co), followed by streptavidin/peroxidase (BD PharMingen) and $\mathrm{H}_{2} \mathrm{O}_{2}$ /orthophenylenediamine substrate (Merck, White House Station, NJ) and read with a 480 -nm filter.

Total IgE level were measured with high-binding microassay plates (Costar) coated with $4 \mu \mathrm{g} / \mathrm{mL}$ of an anti-human $\operatorname{IgE}$ antibody (BD PharMingen) overnight at $4^{\circ} \mathrm{C}$. Plates were blocked with PBS containing $10 \%$ FBS and $0.05 \%$ Tween 20 (Sigma) overnight at $4^{\circ} \mathrm{C}$. Samples were diluted 1:10 in PBS containing 5\% FBS and 0.05\% Tween 20 and incubated overnight at $4^{\circ} \mathrm{C}$. Plates were incubated with biotinylated anti-human $\operatorname{IgE}$ (Sigma), followed by streptavidin/peroxidase (BD PharMingen) and $\mathrm{H}_{2} \mathrm{O}_{2}$ /orthophenylenediamine substrate (Merck) and read with a 480-nm filter. A serum pool from parasite-infected patients was used as a positive control. Umbilical cord serum from a newborn of a nonatopic and nonparasitized mother was used as a negative control.

The assay cutoff for total IgE was determined as the median plus the semi-interquartile deviation of negative controls (sera from persons with 3 negative stool samples collected serially, specific $\operatorname{IgE}<0.35 \mathrm{kU} / \mathrm{L}$, and eosinophils $<2 \%$ in peripheral blood). The assay cutoff for $\mathrm{IgG}_{4}$ for $\mathrm{A}$ lumbricoides was determined as the mean plus an SD of negative controls (sera from children with 3 negative stool samples collected serially). The cutoff for Ascaris species-specific IgE was $0.35 \mathrm{kU} / \mathrm{L}$ or greater, as recommended by the manufacturer. Antibody levels of anti-A lumbricoides $\mathrm{IgG}_{4}$ and total $\operatorname{IgE}$ were defined as positive or negative by using the above cutoffs.

\section{Statistical analyses}

Analyses were conducted for genetic associations by using logistic regression (additive model, except for SNPs with a minor allele frequency $<0.1$, in which we used the dominant model because of zero cells), including sex, age, helminth infection, and covariates, where appropriate. In addition, the first 2 principal components delineated through Eigenstrat on 269 ancestry informative markers were included in the model to address the potential effects of population stratification. For continuous data, such as IL-10 levels and egg burden, analyses were conducted by using linear regression adjusted by sex and age. Both analyses were performed considering adaptive permutations. Permutation procedures provide a computationally intensive approach to generating significance levels empirically. Such values have desirable properties, such as relaxing assumptions about normality of continuous phenotypes and Hardy-Weinberg equilibrium, dealing with rare alleles and small sample sizes, providing a framework for correction for multiple testing, and controlling for identified substructure or familial relationships by permuting only within a cluster. In the case of adaptive permutations, we give up permuting SNPs that are clearly going to be nonsignificant more quickly than SNPs that look interesting. ${ }^{15}$

All genetic analyses were performed with PLINK, and all the graphs were created with STATA 8.2 software (StataCorp, College Station, Tex). We also have explored the relationship between helminth infection and IL10 SNPs and atopic asthma by using causal diagrams (directed acyclic graphs [DAGs]), a powerful tool in epidemiologic research to help answer causal queries, ${ }^{16}$ with DAGitty version 1.1.

\section{RESULTS}

\section{Description of the study population}

Table II summarizes the clinical characteristics of the study population. Atopic wheeze prevalence was $10.72 \%$, and nonatopic wheeze prevalence was $11.69 \%$. We observed greater proportions of children with atopic and nonatopic wheeze in the younger group $(<5$ years old); however, no difference according to sex was found. Markers of allergy, such as SPT reactivity (66.9\%), specific IgE levels to common aeroallergens (100\%), and total IgE levels $(1.62 \mathrm{kU} / \mathrm{mL})$, were greater in the group with atopic wheeze.

\section{IL10 genetic variants and IL-10 levels}

Table III summarizes significant associations observed between IL10 polymorphisms and IL-10 levels in supernatant of cultured blood cells after A lumbricoides stimulation. The $\mathrm{G}$ allele of exonic marker rs3024496 was negatively associated with IL-10 production $(P=.05)$. Association results of all genotyped variants can be found in Table E1 in this article's Online Repository at www.jacionline.org. Functional data on IL-10 levels according to the genotype of rs3024496 is presented in Fig 2, showing that subjects with the wild-type G allele are less likely to produce IL-10 in whole-blood cultures.

\section{IL10 genetic variants and helminth infection}

Significant associations between ILIO genetic variants and A lumbricoides and $T$ trichiura infections are summarized in Table IV. Marker rs3024498 (C allele) was negatively associated with $T$ trichiura current $(P=.04)$ and chronic $(P=.0003)$ infection, coinfection with A lumbricoides and $T$ trichiura $(P=.04)$, and markers of infection, including $\operatorname{IgE}(P=.01)$ and $\operatorname{IgG}_{4}$ anti-A lumbricoides $(P=.004)$ levels. Two other variants were associated with A lumbricoides chronic infection (rs1800896, $P=.04)$ and IgE anti-A lumbricoides (rs1554286, $P=.02$ ). 


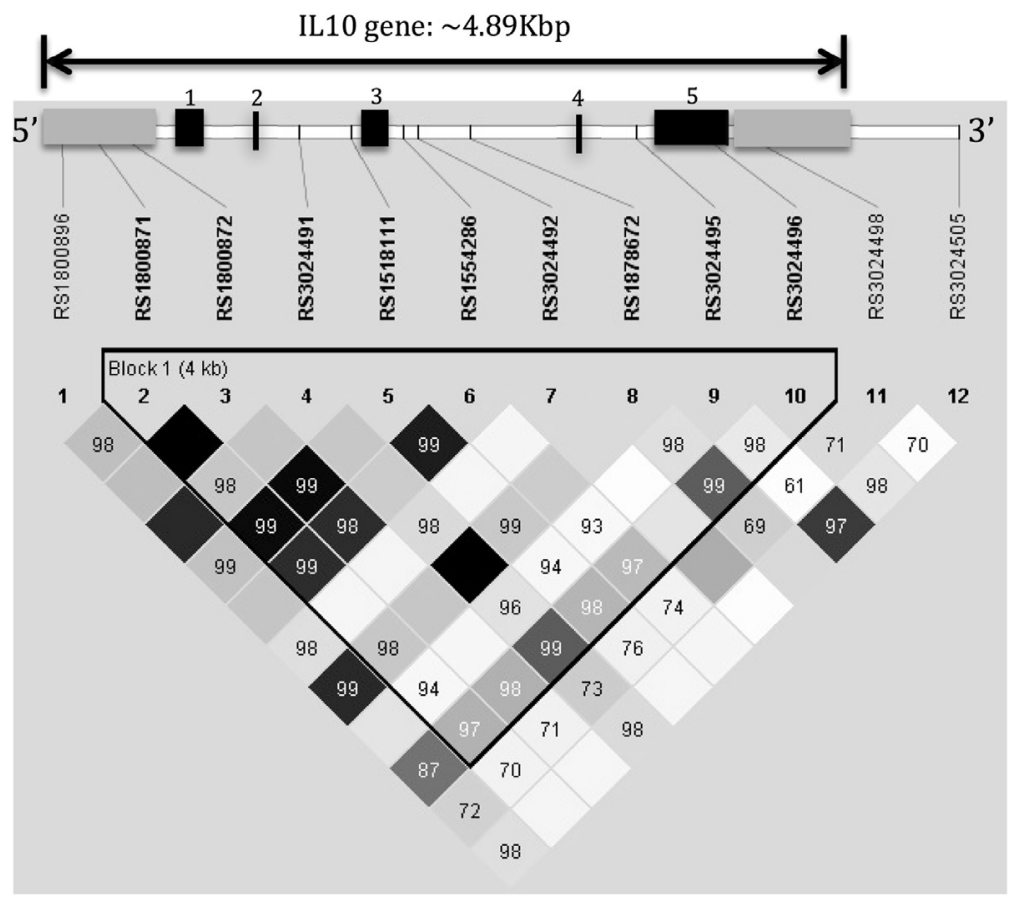

FIG 1. Pairwise LD within Haploview by using the $R^{\prime}$ squared statistic for the $I L 10$ gene. Intensity of shading indicates the degree of confidence in the $\mathrm{R}^{\prime}$ value. Dark solid squares indicate an $\mathrm{R}^{\prime}$ value of 1 . Untranslated regions are indicated by gray bars, exons by black bars, and introns by white bars. Exons are numbered from $5^{\prime}$ to $3^{\prime}$.

TABLE II. Characteristics of the Social Changes Asthma and Allergy in Latin America population according to asthma status and variables included in this study

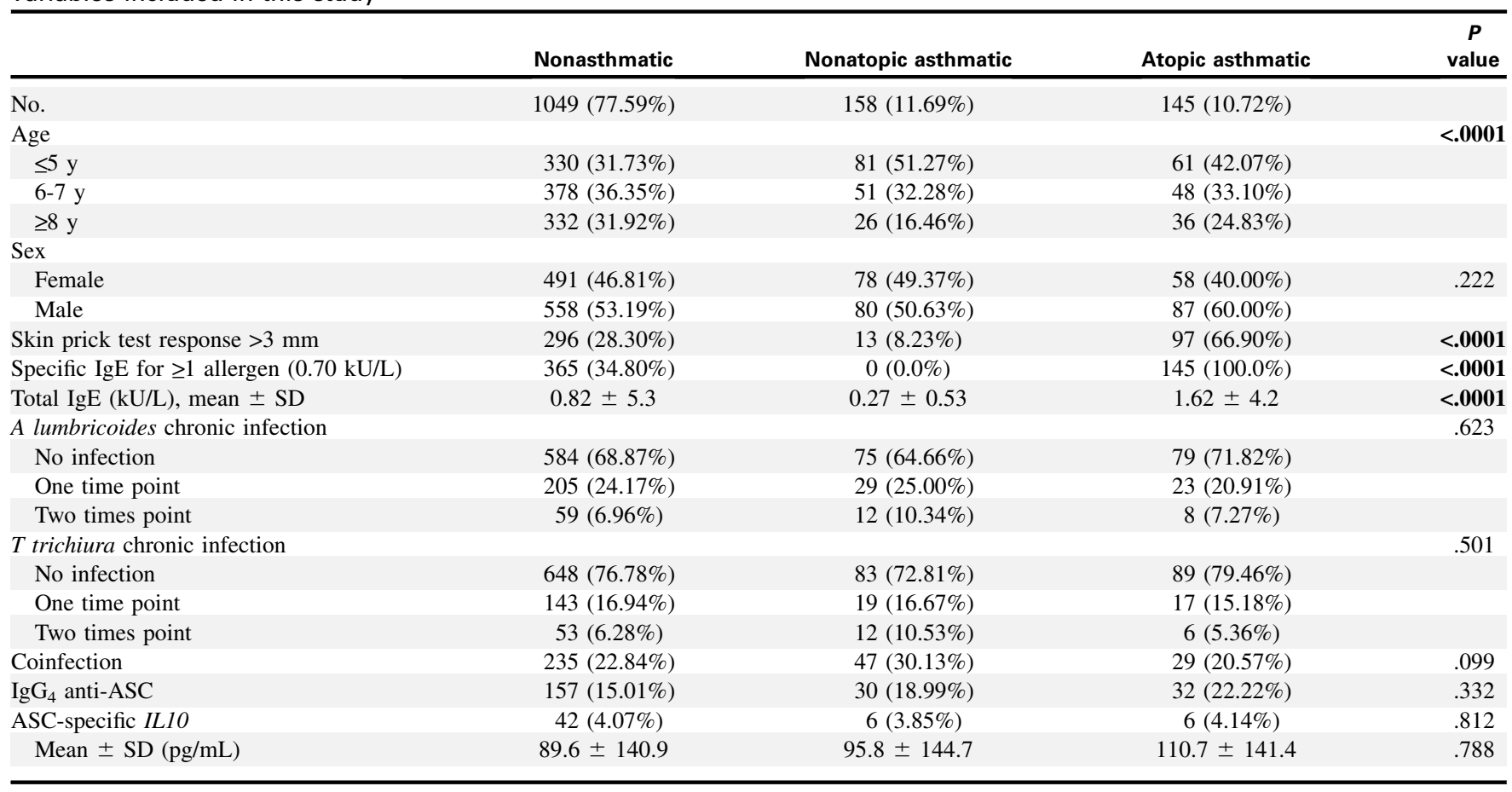

Statistically significant values are shown in boldface. ASC, Ascaris lumbricoides.

Additional IL10 variants, notably rs 1518111 (T allele), rs 1800872 ( $\mathrm{T}$ allele), and rs1800871 (A allele), were also associated with lower levels of $T$ trichiura eggs per gram of stool (data not shown). A complete list of all SNPs included in this study is presented in Tables E2 to E7 in this article's Online Repository at www.jacionline.org. 
TABLE III. Significant association between IL10 SNPs and levels of IL-10 production under $A$ lumbricoides stimulation in wholeblood culture by using linear regression adjusted by sex and age

\begin{tabular}{|c|c|c|c|c|c|c|c|c|c|}
\hline Marker & Chromosome & Base pairs & Allele & MAF & No. & $\boldsymbol{\beta}$ & Cl & $P$ value & EMP1 \\
\hline rs3024496 & 1 & 206941864 & $\mathrm{G}$ & 0.38 & 99 & -0.52 & -1.00 to -0.04 & .038 & 0.037 \\
\hline
\end{tabular}

Statistically significant values are shown in boldface. $E M P 1, P$ value considering adaptive permutations by using the additive model; $M A F$, minor allele frequency.

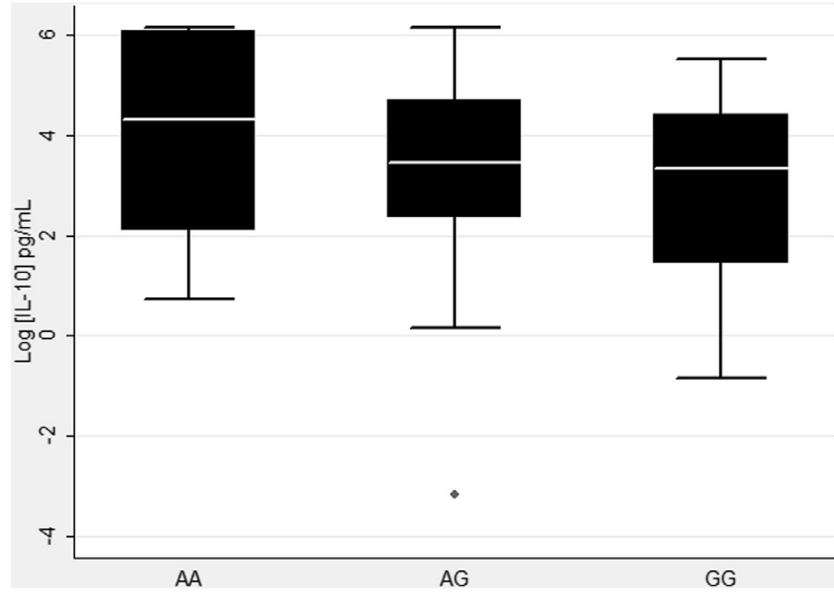

FIG 2. Functional analysis of IL-10 production according to the genotype for rs3024496. Box plot for IL-10 production for AA, AG, and GG genotypes for SNP rs3024496. Analysis is adjusted for sex, age, and principal components for ancestry informative markers $(P=.05)$.

\section{IL10 genetic variants and allergy}

Table V summarizes significant associations between IL10 SNPs, wheeze, and markers of allergy. The most consistent marker, rs3024492, was positively associated with atopic wheeze $(P=.01)$, total IgE level $(P=.008)$, and SPT response to cockroach $(P \leq .01)$. In addition, 2 other markers, rs3024505 $(P=.01)$ and $\mathrm{rs} 3024495(P=.03)$, were positively associated with specific IgE levels to $D$ pteronyssinus. A negative association was observed between specific IgE levels to $B$ germanica and rs1800872 (allele T, $P=.04$ ). A list of all variants included in this study can be found in Tables E8 to E13 in this article's Online Repository at www.jacionline.org. In Fig 3 the DAG summarizes relationships between all the variables observed in the study.

\section{DISCUSSION}

We have demonstrated that $I L 10$ genetic variants are negatively associated with IL-10 production and helminth infection and, conversely, are positively associated with risk of atopic wheeze and markers of allergy (ie, SPT response and allergen-specific IgE level). This observation can explain, at least in part, the growing body of evidence that associates the exposure to helminthic parasites with protection against allergic disease.

The human immune response to helminth infections is associated with increased IgE levels, tissue eosinophilia and mastocytosis, and the presence of $\mathrm{CD}^{+} \mathrm{T}$ cells that preferentially produce IL-4, IL-5, and IL-13. ${ }^{17} \mathrm{~T}_{\mathrm{H}}$ 2-mediated mechanisms are considered to mediate protective immunity against these extracellular parasites. ${ }^{18}$ Parasites in the tissues stimulate a strong localized $\mathrm{T}_{\mathrm{H}} 2$ response characterized by an eosinophil-rich inflammatory infiltrate. ${ }^{19}$ However, especially during chronic infections, the activation of a regulatory network induced by helminths has been also suggested to be responsible for preventing the elimination of the worms but also protection of the host from immunopathology that would otherwise result from excessive inflammation. ${ }^{3,20}$ A popular explanation for the increase in the prevalence of allergy is the hygiene hypothesis, which attributes the allergy epidemic to a failure to develop appropriate immune regulation because of reduced exposures to microbes and their products in childhood, including helminths. ${ }^{21}$

We have previously demonstrated in the same Social Changes Asthma and Allergy in Latin America population that children who live in areas without "hygiene" (ie, lack of garbage collection or sewage systems) ${ }^{7}$ or who are chronically infected with helminths ${ }^{5}$ are more likely to produce IL-10 in culture compared with children living in cleaner living conditions or who are not infected with helminths. Given these observations, we wished to address whether the IL-10 upregulation previously demonstrated in this cohort is induced solely by relevant environmental exposures (ie, infection) or whether a genetic component is also involved.

From a total of 12 variants included in this study, the $G$ allele of marker rs3024496 was negatively associated with IL-10 production on A lumbricoides stimulation, as demonstrated also by functional analysis in peripheral blood cells. The same variant was previously associated with IgE levels in a close population in Brazil (Bahia, Brazil, as well). This was a family-based study, and we have identified some replicated SNPs; for example, rs3024496 was associated with IgE levels in this population. ${ }^{22}$ In another study in Costa Rica, the same SNP was also associated with IgE levels in a population with high exposure to house dust mite and with increased asthma risk. ${ }^{23}$ In our study we did not observe any direct association between the $\mathrm{G}$ allele of rs3024496 and infection or allergy markers; however, this marker is in high LD with markers that were associated with allergy. Thus might be explained by the tendency of producing less IL-10 in subjects who carry this allele because IL-10 was previously associated with suppressed production of IgE and effector cell activities, such as those from mast cells and eosinophils. ${ }^{24}$ The SNP rs3024496 is located $117 \mathrm{bp}$ after the translation stop codon and probably does not alter the protein's structure. The functionality of the protein should not be changed given the location of the SNP. SNPs in the 3' untranslated region can influence the stability of mRNA, however, which might have an effect on protein levels in the cell (the more stable the mRNA, the higher the concentrations of protein).

Marker rs3024498 (C allele) was highly associated with helminth infection, especially chronic $T$ trichiura infection. The $\mathrm{C}$ allele of rs3024498 was also negatively associated with levels of specific $\mathrm{IgE}$ (current infection) and $\mathrm{IgG}_{4}$ (past infection) to $A$ lumbricoides. A negative association was also found for the A allele of rs3024505 with coinfection by $A$ lumbricoides and $T$ trichiura. No previous studies have associated the ILIO marker rs3024498 with helminth infection or asthma; however, this marker was determined to be a tag SNP for both the CEU and YRI populations (included in this study as reference populations 
TABLE IV. Significant association between IL 10 SNPs and helminth chronic infection, current infection, and coinfection for A lumbricoides and $T$ trichiura by using logistic regression adjusted by sex and age

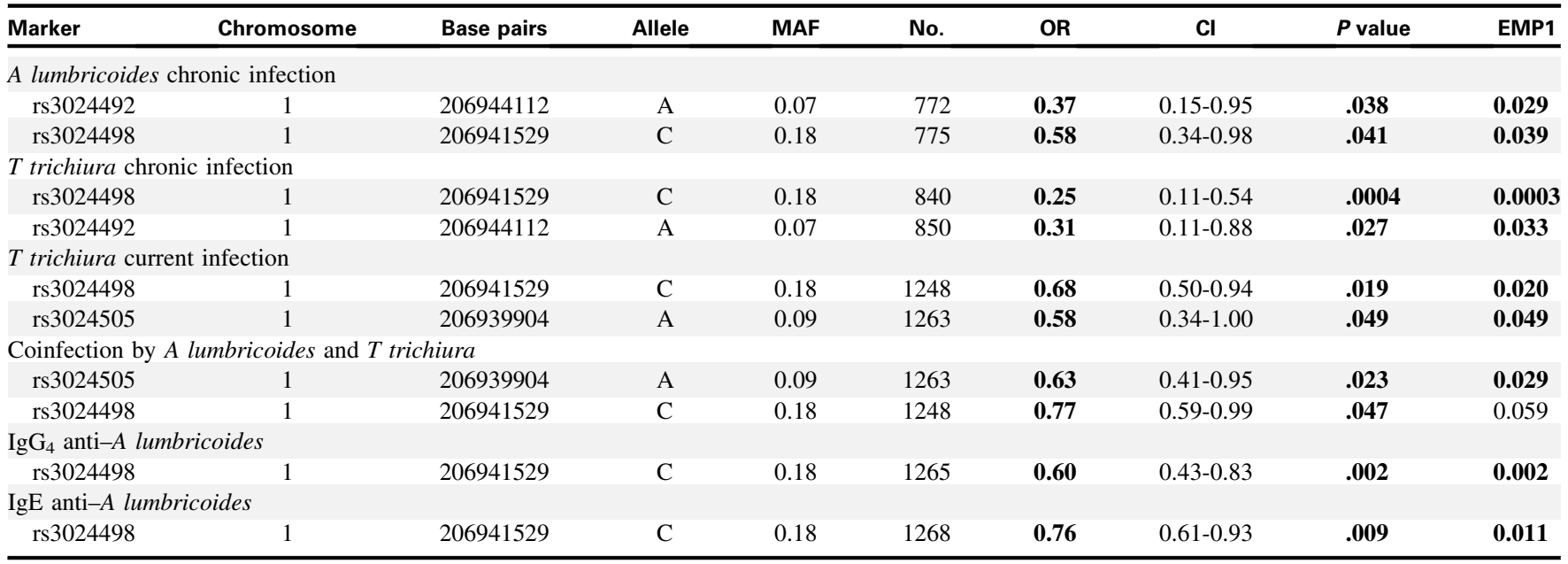

Statistically significant values are shown in boldface. $H W E$, Hardy-Weinberg equilibrium; $M A F$, minor allele frequency; $O R$, odds ratio.

TABLE V. Significant association between IL10 SNPs and atopic asthma, total and specific lgE levels, and SPT responses by using logistic regression adjusted by sex, age, and helminth infection

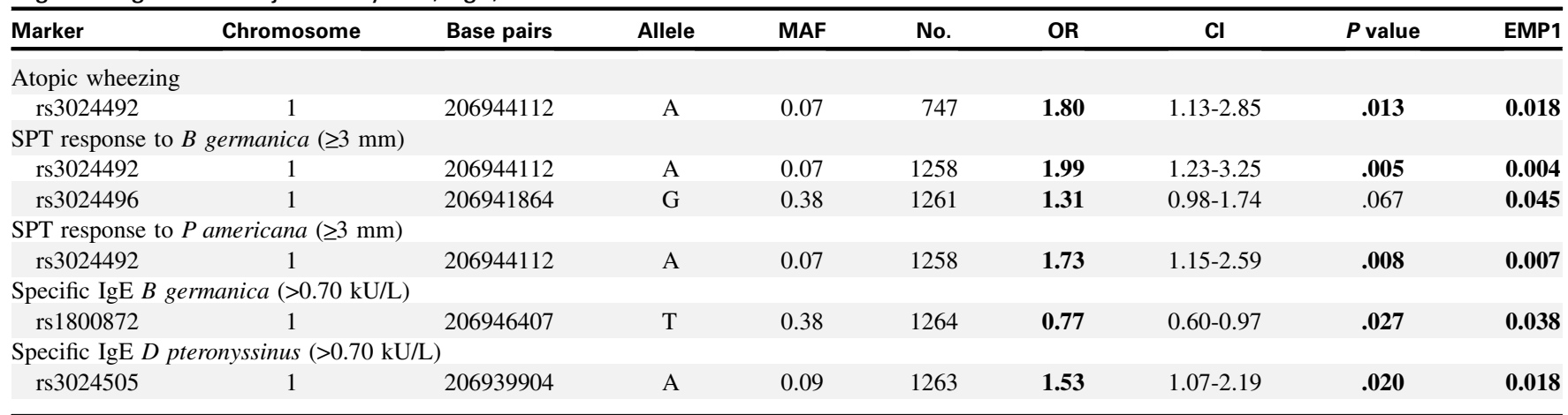

Statistically significant values are shown in boldface. $H W E$, Hardy-Weinberg equilibrium; $M A F$, minor allele frequency; $O R$, odds ratio.

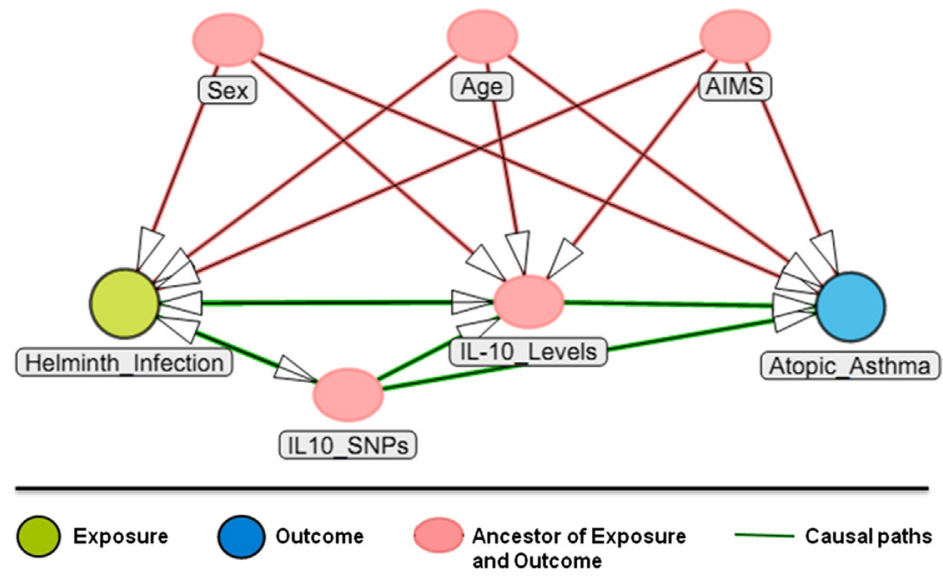

FIG 3. DAG showing 2 causal paths between helminth infection and atopic asthma. The relationships observed were adjusted for sex, age, and ancestry informative markers.

to capture the European and African admixture characteristic of the Brazilian population). Additional IL10 variants, notably rs1518111 (T allele), rs1800872 (T allele), and rs1800871 (A allele), were also associated with lower numbers of T trichiura eggs per gram of stool. Markers rs 1800872 and rs1800871 were previously described by our group to be associated with high total IgE levels in a Brazilian population living in a region endemic for Schistosoma mansoni. ${ }^{22}$ Marker rs3025405 (downstream gene) 
was previously described to be associated with the reduction in IL-10 levels in healthy and tuberculosis-infected subjects. ${ }^{25} \mathrm{Al}-$ though not in the same LD block as the majority of SNPs studied herein, marker rs3024505 is in strong LD with marker rs3024496, which was related to lower IL-10 levels in this study. Thus the signal observed for rs 3024505 might be related to the exomic variant rs3024496. Finally, marker rs3024505 was associated with increased IgE levels on high exposure to mite, ${ }^{23}$ which again might be related to suppression of IL-10 production.

We have previously demonstrated that children chronically infected by $T$ trichiura are more likely to produce IL-10 in culture when peripheral blood cells were stimulated with A lumbricoides antigen. ${ }^{5}$ To verify the gene environment interaction between $T$ trichiura infection, IL-10 production, and IL10 SNPs, we fit a logistic regression model in which we found that the infection is positively associated with IL-10 production, even after adjustment with the $\mathrm{G}$ allele for rs3024496, which was described early to protect against IL-10 production. In addition, we did not find a significant interaction effect between the infection and the $\mathrm{G}$ allele of rs3024496 on IL-10 production ( $P=.72$, data not shown). Thus our results do not support this effect modification on the probability of producing IL-10. One possible explanation is that the SNPs related to suppress IL-10 production are not the same that protect against infection. On the basis of these results, we could also speculate that the genotype cannot predict the production of a protein and that the exposure to environmental factors can modulate this production. Further studies specifically designed to focus on the gene-environment interaction must be conducted to confirm these findings.

Regarding allergy markers, the most consistent variant associated with atopic wheezing and markers of allergy in this study was rs3024492. The A allele of this variant (rs3024492), which is in high LD with rs3024496 (associated with decreasing IL-10 levels), was positively associated with atopic wheeze (but not nonatopic wheeze) and a positive SPT response to cockroach ( $B$ germanica and $P$ americana). Marker rs3024505 (in high LD with rs3024496) was positively associated with specific IgE to D pteronyssinus. Marker rs1800872 (negatively associated with $T$ trichiura eggs in stool) was negatively associated with specific IgE to $B$ germanica. As mentioned above, in contrast to these results, marker rs 1800872 was also described by our group to be associated with high total IgE levels. ${ }^{22}$ These conflicting results are currently being explored to better elucidate the role of rs 1800872 in $\mathrm{IgE}$ production.

Of note is the observation that nonatopic wheeze constitutes the majority of asthma cases in the Social Changes Asthma and Allergy in Latin America population and that perhaps the mechanism proposed herein can be applied to atopic wheeze only. To further investigate this conundrum, additional studies conducted in populations in which nonatopic asthma predominates are needed to elucidate other variants that might contribute to asthma risk in such unique populations.

Previous work addressing the role of human genes that modulate susceptibility to helminths ${ }^{26}$ demonstrated that the IL10 SNP rs1554286 is associated with both the diversity of helminth species that are transmitted in different geographic regions and the pathogenesis of atopic conditions or airway hyperresponsiveness. This marker was included in our study and was found to be in high LD with several markers that were coassociated with allergic markers and helminth infections in the genome-wide search by Fumagalli et al. ${ }^{26}$
In the present study we found that SNPs in the ILIO gene were strongly associated with helminth infection and allergy markers, even after adjusting for ancestry informative markers. From these observations, we hypothesize that changes in exposure to helminths might explain, at least in part, the occurrence of allergic diseases in tropical populations. Fig 3 shows the DAG showing 2 causal paths between helminth infection and atopic asthma proposed in the present study. At this time, we cannot determine whether a specific ILIO variant or several variants are responsible for the reduction in IL-10 levels, helminth infection, and the increasing risk of atopic wheeze because the majority of markers genotyped are in high LD. Ongoing sequencing studies might elucidate the true underlying causal variant or variants within this locus identified by using a tag SNP approach.

We acknowledge the funding agencies that have supported this work and technical assistance from Pat Oldewurtel.

\section{Key messages}

- IL10 genetic variants that downregulate IL-10 production on $\boldsymbol{A}$ lumbricoides stimulation are negatively associated with helminth infection and positively associated with allergy.

- IL10 genetic variants might play a role in helminth infection and allergy; we hypothesize that diminished exposure to helminths might explain, at least in part, the occurrence of allergic diseases in tropical populations.

\section{REFERENCES}

1. Bach JF. The effect of infections on susceptibility to autoimmune and allergic diseases. N Engl J Med 2002;347:911-20.

2. Rook GA. The hygiene hypothesis and the increasing prevalence of chronic inflammatory disorders. Trans R Soc Trop Med Hyg 2007;101:1072-4.

3. Maizels RM, Yazdanbakhsh M. Immune regulation by helminth parasites: cellular and molecular mechanisms. Nat Rev Immunol 2003;3:733-44.

4. Guarner F, Bourdet-Sicard R, Brandtzaeg P, Gill HS, McGuirk P, van Eden W, et al. Mechanisms of disease: the hygiene hypothesis revisited. Nat Clin Pract Gastroenterol Hepatol 2006;3:275-84.

5. Figueiredo CA, Barreto ML, Rodrigues LC, Cooper PJ, Silva NB, Amorim LD, et al. Chronic intestinal helminth infections are associated with immune hyporesponsiveness and induction of a regulatory network. Infect Immun 2010;78:3160-7.

6. Figueiredo CA, Alcantara-Neves NM, Amorim LD, Silva NB, Carvalho LC, Cooper PJ, et al. Evidence for a modulatory effect of IL-10 on both Th1 and Th2 cytokine production: the role of the environment. Clin Immunol 2011;139:57-64.

7. Figueiredo C, Alcântara-Neves N, Veiga R, Amorim L, Dattoli V, Mendonça L, et al. Spontaneous cytokine production in children according to biological charac teristics and environmental exposures. Environ Health Perspect 2009;117:845-9.

8. Barreto ML, Cunha SS, Alcântara-Neves N, Carvalho LP, Cruz AA, Stein RT, et al. Risk factors and immunological pathways for asthma and other allergic diseases in children: background and methodology of a longitudinal study in a large urban center in Northeastern Brazil (Salvador-SCAALA study). BMC Pulm Med 2006; $6: 15$.

9. Rodrigues LC, Newcombe PJ, Cunha SS, Alcantara-Neves NM, Genser B, Cruz AA, et al. Early infection with Trichuris trichiura and allergen skin test reactivity in later childhood. Clin Exp Allergy 2008;38:1769-77.

10. Barreto M, Genser B, Strina A, Teixeira M, Assis A, Rego R, et al. Impact of a citywide sanitation programme in Northeast Brazil on intestinal parasites infection in young children. Environ Health Perspect 2010;118:1637-42.

11. Livak KJ. Allelic discrimination using fluorogenic probes and the 5' nuclease assay. Genet Anal 1999;14:143-9.

12. Alcantara-Neves NM, Veiga RV, Dattoli VC, Fiaccone RL, Esquivel R, Cruz Á, et al. The effect of single and multiple infections on atopy and wheezing in children. J Allergy Clin Immunol 2012;129:359-67, e1-3. 
13. Hoffman WA, Pons JA, Janer JL. The sedimentation concentration method in Schistosomiasis mansoni. Puerto Rico J Public Health 1934;9:281-98.

14. Katz N, Chaves A, Pellegrino J. A simple device for quantitative stool thick-smear technique in Schistosomiasis mansoni. Rev Inst Med Trop Sao Paulo 1972;14: 397-400.

15. Purcell S, Neale B, Todd-Brown K, Thomas L, Ferreira MA, Bender D, et al. PLINK: a tool set for whole-genome association and population-based linkage analyses. Am J Hum Genet 2007;81:559-75.

16. Shrier I, Platt RW. Reducing bias through directed acyclic graphs. BMC Med Res Methodol 2008;8:70

17. Fallon PG, Mangan NE. Suppression of TH2-type allergic reactions by helminth infection. Nat Rev Immunol 2007;7:220-30.

18. Anthony RM, Rutitzky LI, Urban JF, Stadecker MJ, Gause WC. Protective immune mechanisms in helminth infection. Nat Rev Immunol 2007;7:975-87.

19. Cooper PJ. Interactions between helminth parasites and allergy. Curr Opin Allergy Clin Immunol 2009;9:29-37.

20. Wilson MS, Taylor MD, Balic A, Finney CA, Lamb JR, Maizels RM. Suppression of allergic airway inflammation by helminth-induced regulatory T cells. J Exp Med 2005;202:1199-212.
21. Garn H, Renz H. Epidemiological and immunological evidence for the hygiene hypothesis. Immunobiology 2007;212:441-52

22. Grant AV, Araujo MI, Ponte EV, Oliveira RR, Cruz AA, Barnes KC, et al. Polymorphisms in IL10 are associated with total immunoglobulin E levels and Schistosoma mansoni infection intensity in a Brazilian population. Genes Immun 2011;12: 46-50.

23. Hunninghake GM, Soto-Quirós ME, Lasky-Su J, Avila L, Ly NP, Liang C, et al. Dust mite exposure modifies the effect of functional IL10 polymorphisms on allergy and asthma exacerbations. J Allergy Clin Immunol 2008;122:93-8, e1-5.

24. Akdis M. T-cell tolerance to inhaled allergens: mechanisms and therapeutic approaches. Expert Opin Biol Ther 2008;8:769-77.

25. Abhimanyu Mangangcha IR, Jha P, Arora K, Mukerji M, Banavaliker JN, et al. Differential serum cytokine levels are associated with cytokine gene polymorphisms in north Indians with active pulmonary tuberculosis. Infect Genet Evol 2011;11:1015-22.

26. Fumagalli M, Pozzoli U, Cagliani R, Comi GP, Bresolin N, Clerici M, et al. The landscape of human genes involved in the immune response to parasitic worms. BMC Evol Biol 2010;10:264. 